\title{
Application of a magnetic mass spectrometer to ionization studies in impure shock-heated argon
}

\author{
By B. STURTEVANT \\ Graduate Aeronautical Laboratories, California Institute of Technology, \\ Pasadena, California
}

(Received 15 October 1965)

\begin{abstract}
A study of the unique role of impurities in the initial stages of ionization relaxation in shock-heated argon, using a sampling mass spectrometer to determine the ionic products of the reaction, is described. 'The ions are extracted from the shock tube through a small orifice in the end wall after they have diffused through the dense thermal layer adjacent to the wall from the ionizing gas behind the reflected shock wave. The ion diffusion is analysed in detail to assess the possibility that the sampling process alters the reaction products. It is shown that this is unlikely because the impurities are in dilute concentration and the reaction is studied in its initial stages. This mode of sampling is compared with others.

The experiments were conducted in argon at temperature of $16,600^{\circ} \mathrm{K}$ and pressure of $16 \mathrm{mmHg}$ with an estimated impurity level of 300 parts per million. A surprisingly large number of different ions were detected during the initial stages of ionization. $\mathrm{O}^{+}$and $\mathrm{H}^{+}$were found in much greater amounts than any of the other products, each being about five times more abundant than $\mathrm{A}^{+}$. The results suggest that $\mathrm{H}_{2} \mathrm{O}$ is probably quite generally the most important impurity in thermal-ionization experiments, and that ionization 'incubation' is due to dissociation of molecular impurities (especially $\mathrm{H}_{2} \mathrm{O}$ ) before ionization commences. Possible explanations of the well-known efficiency of small amounts of impurities in initiating ionization are discussed.
\end{abstract}

\section{Introduction}

An important feature of an ionizing shock wave is the non-uniform region behind the shock, where ionization relaxation takes place; if the shock is to be used to generate a uniformly ionized gas for, say, experiments in plasma physics, it is important to be able to predict the extent of the non-uniform region, or the relaxation time. Early studies of the thermal ionization of argon (Petschek \& Byron 1957) showed that the relaxation time is determined by the chemical kinetics of the initial stages of the relaxation process. This is because the initial stages are inherently slower than the later stages due to the fact that the inelastic atom-atom collisions that initiate ionization are much less efficient than the electron-atom collisions that dominate the final approach to equilibrium. It was also discovered in early studies that the relaxation time (i.e. the rate of ionization in the initial stages) depends strongly on the presence of impurities, even for impurity levels as low as a few parts per million. The explanation of this unique 
behaviour is presently not known and, as a consequence, it is impossible to predict the relaxation time in any given experiment with better than order-ofmagnitude accuracy.

Progress has recently been made in the understanding of the ionization of pure monatomic gases by Harwell \& Jahn (1964), who demonstrated that the rate of electron production in the initial stages can be explained by a two-step atom-atom collisional process in which the first step, excitation of the atom to its resonance state, is rate-determining (depopulation of the excited state by radiative decay to the ground state is negligible presumably because of resonance-radiation trapping), and the second step, ionization of the excited atom, is very fast. However, the purity levels at which this mechanism dominates are so high that it is unlikely that it determines the relaxation time in the majority of experiments in ionized gases, in which extreme precautions to ensure high purity, such as hightemperature bake-out of the shock tube, are not usually taken.

Since research-grade gases of high purity are readily available and are frequently used, the major source of impurities in ionization experiments is usually leakage and degassing into the shock tube after introduction of the test gas prior to the experiment. Therefore, contamination generally consists of air, water vapour, hydrocarbons from pump oils, and carbon dioxide. How one or more of these gases in such small concentrations can so importantly affect ionization is now the central question in the problem of ionization relaxation in the rare gases.

As a first step toward answering this question, the ionic products in the initial stages of the ionization reaction in 'impure' argon have been determined experimentally. For this purpose a magnetic mass spectrometer has been used to analyse the ionizing gas between the reflected shock wave and the end wall of the GALCIT 17 in. diameter shock tube (Liepmann, Roshko, Coles \& Sturtevant 1962). The use of sampling mass spectrometers in studies of fast reactions in shock tubes is a relatively new technique (Bradley \& Kistiakowsky 1961). Its utility derives primarily from the ability of the spectrometer to identify the individual components of a mixture of gases. It is to take advantage of this capability that the mass spectrometer is used in the present experiments. Partly because of the particular application, and partly because this machine was conceived and designed in 1961-62 independently of other early work in this field, it is somewhat different from the now more frequently used time-of-flight mass spectrometer.

In this study the ionizing gas in the shock tube is the source of ions for the spectrometer, so an electron beam for ionizing the sample is not necessary. Thus, one of the links in the chain of processes that lies between the reaction and the final measurement in sampling experiments has been eliminated. The spectrometer is mounted on the end wall of the shock tube and ions are sampled from the shock-heated gas through small orifices in the end wall. In these experiments the orifice diameter is much smaller than the thickness of the layer of gas adjacent to the wall that is cooled by heat transfer, so the ions diffuse through this dense thermal layer before entering the stream of neutral particles flowing through the orifices. The accelerating electrodes of the mass spectrometer are immediately downstream of the orifices. 
As in all sampling experiments, the question of whether the reaction products are altered during the sampling operation is of primary importance. Consequently, the behaviour of the diffusing ions in the end-wall thermal layer is studied in some detail in $\S 3$. It is shown that, primarily because in the present experiment only the initial stages of the reaction are studied and also because the 'reactive' species (impurities) are in dilute concentration, it is unlikely that the sample is perturbed by the thermal layer.

The analyser is a $6 \mathrm{in}$. radius, $60^{\circ}$ sector magnetic field Nier spectrometer (Nier 1947) with an electron-multiplier collector. During each run the continuous time history of one mass peak is measured with microsecond resolution. In comparison with the more customary technique of measuring the entire spectrum at one or more different times during the run (Bradley \& Kistiakowsky 1961), the present method suffers from the disadvantage of requiring several runs to obtain a complete spectrum but has the advantage of much better time resolution. Indeed, this advantage is crucially important in experiments at high temperatures or low densities, when the effects of the important departures from ideal shock-tube behaviour that occur in these circumstances are difficult to detect and understand even with a continuous recording instrument. Of course this method is only possible if test conditions in the shock tube can be precisely repeated time after time; the GALCIT 17 in. shock tube, with its unique diaphragm-opening mechanism, is well suited for such an application.

Just before a run, the spectrometer is set on the mass peak of interest using the removable Nier-type electron-beam ion source described in $\$ 2$. Because of space requirements for this source the orifices are not in the end plate of the 17 in. shock tube but are some distance downstream, in the end of a $3 \mathrm{in}$. diameter tube (the 'cookie cutter') that extends upstream into the shock tube and cuts a piece from the centre of the shock wave.

In $\S 4$ the first results of an investigation of the role of impurities in the initial stages of ionization in 'impure' argon are reported. The first $200 \mu$ sec of an estimated $2 \mathrm{msec}$ long relaxation process at $16,600^{\circ} \mathrm{K}$ is studied. After an incubation period of 30-50 $\mu \mathrm{sec}$ a surprisingly large number of different ionic products are observed, but by far the most abundant are ionized atomic oxygen and hydrogen. It is suggested that during the incubation period dissociation of water vapour takes place, after which the most efficient mechanism for producing ions is $(a)$ multistep ionization of $\mathrm{O}$ with metastable excited states acting as intermediates, and $(b)$ ionization of $\mathbf{H}$ by accidentally resonant charge exchange with $\mathrm{O}^{+}$.

\section{Mass spectrometer}

\subsection{General}

The spectrometer (figure 1) is similar to the one described by Nier (1947); indeed, the arrangement of the slits, their spacing and dimensions, the electron-beam source, and the magnet pole pieces and gap are identical. The envelope is all-metal (stainless steel) and the flanged joints are sealed by neoprene $O$-rings. A 4 in. oil diffusion pump, isolated from the spectrometer by a straight-through liquidnitrogen cold trap of our own design, pumps the non-bakeable system to about 
$10^{-6} \mathrm{mmHg}$. It is sufficiently large to maintain a pressure of $7 \times 10^{-5} \mathrm{mmHg}$ in the spectrometer when the shock tube containing argon at $30 \mu \mathrm{Hg}$ is connected to the spectrometer through the sampling orifices (thirty 0.007 in. diameter holes in a double row aligned with the first slit of the spectrometer).

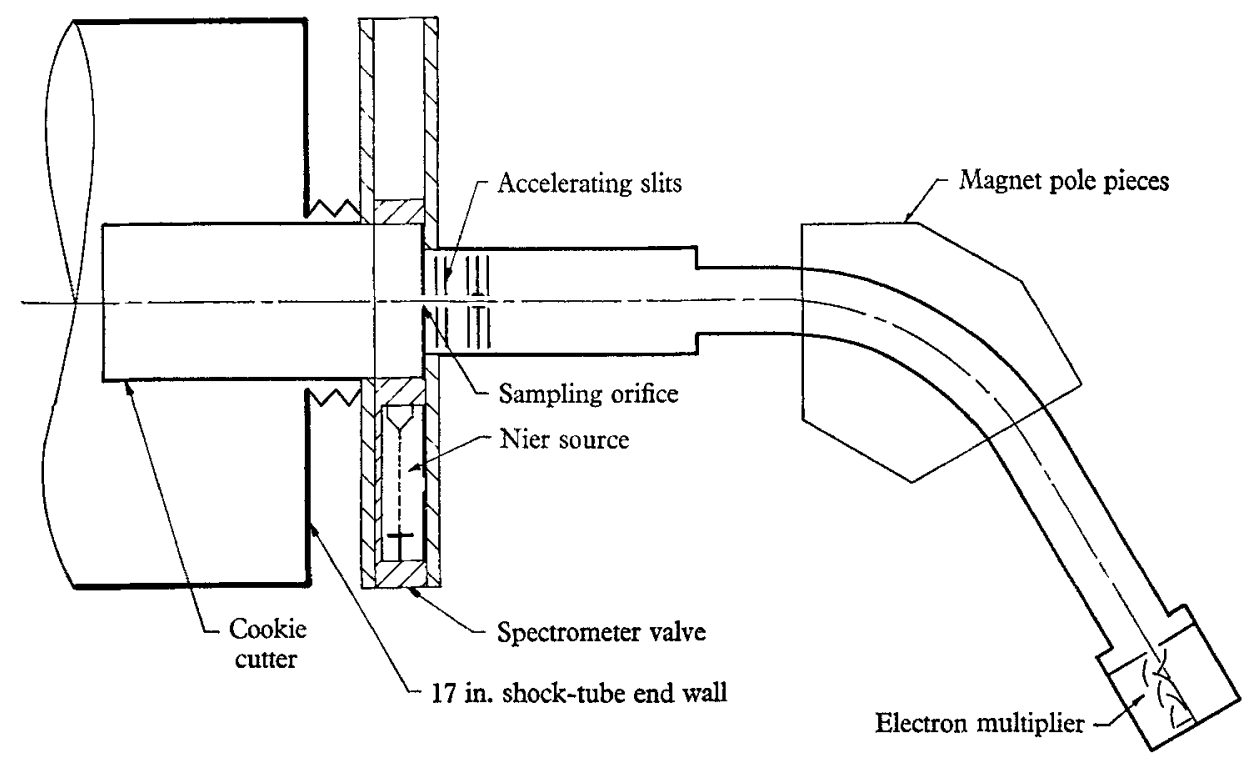

Figure: 1. The mass spectrometer installation.

The electronic circuitry required for the time-resolved study of one mass peak is considerably less sophisticated than that required for a scanning instrument. This, combined with the simple construction of many of the components of a magnetic spectrometer, results in an installation that can be built by the experimenter for about one-fifth the cost of a commercially available time-of-flight spectrometer. Of course the magnetic spectrometer with its large magnet is much heavier and more bulky than a time-of-flight spectrometer, but usually this is not a consideration in shock-tube experiments.

This spectrometer is unique in that its source (the shock tube) is necessarily at ground potential. Consequently, the envelope and collector are isolated electrically from the pump, magnet, supports, etc., and are biased at $-2200 \mathrm{~V}$, the ionaccelerating voltage. The same voltage is used for the electron multiplier in the collector, so the anode of the multiplier is, conveniently enough, at ground potential.

Power supplies are similar to those of Nier's earlier machine (Nier 1940). The source filament is powered by a $12 \mathrm{~V}$ wet-cell battery, as is the magnet. The accelerating and electron-multiplier voltage is supplied by twenty-four $90 \mathrm{~V}$ drycell batteries. The two magnet coils have 2200 turns of 12-gauge magnet wire each. This arrangement results in an instrument that, once having stabilized on a peak (typically after a few minutes), shows no perceptible drift from that peak for as much as half an hour. 


\subsection{Source}

One unique feature of the mechanical design of this instrument is its removable electron-beam source, which is used to set the spectrometer on the desired mass peak just before a run. As indicated in figure 1, the Nier source is housed in a rectangular block (the 'spectrometer valve') that slides between two fixed plates. When the valve is 'closed' the source slit is aligned with the draw-out slit and the shock tube is not connected to the spectrometer. In the 'open' position, shown in figure 1, the source has been moved aside and in its place is a 3 in. diameter hole aligned with the 'cookie-cutter' extension of the 17 in. shock tube. A thin metal diaphragm containing the sampling orifices closes the end of this hole and forms the end wall of the shock tube in the experiments. The cookie cutter and the spectrometer are shock isolated from the shock tube by a metal bellows connexion.

The spectrometer valve is pneumatically actuated. A few milliseconds after each run the gate signal from an oscilloscope starts the valve closing and turns off the high voltage. The source is pumped independently of the rest of the spectrometer through a $\frac{3}{4} \mathrm{in}$. diameter flexible bellows.

\subsection{Collector}

The time response necessary for studies of shock-tube flows is achieved by using a 10-stage electron multiplier $\dagger$ in the collector. It gives a current amplification of 2000 and, when its output is shunted to ground through a $20 \mathrm{k} \Omega$ resistor and the resulting voltage is coupled by a cathode follower to a Tektronix Type $\mathrm{L}$ preamplifier, the input noise current is about $10^{-13} \mathrm{amp}$ and the time response is $1 \mu \mathrm{sec} . \ddagger$

\section{Sampling}

The extent to which the instrumentation in experiments that sample reacting gases perturbs the reactions or reaction products has always been a matter of concern. Because of the variety and difficulty of the fluid-mechanical and chemical processes involved, it is difficult to make any general conclusions about sampling. Generally, the sample is expanded to low temperature and density as rapidly as possible to $(a)$ freeze the reaction under study by reducing the number of energetic collisions, and $(b)$ reduce the probability of other reactions that might alter the composition of the sample and thereby lead to spurious conclusions. In many experiments the gas is expanded to free-molecular conditions for analysis, in which case the total number of collisions an atom experiences at conditions other than those in the reaction vessel is one of the most important factors affecting the chemical changes in the expansion.

The simplest arrangement in shock-tube sampling experiments is that in which gas from behind the reflected shock wave is removed through an orifice or

$\dagger$ DuMont SPM 18-01-401.

$\ddagger$ The response time was measured by pulsing the draw-out plates of the spectrometer with a $-100 \mathrm{~V}$ square wave while the electron-beam source was operating in the standard fashion. The collector output was observed on an oscilloscope. 
nozzle in the end wall (figure 2). In this case the effect of the proximity of the end wall to the sampled gas can be outlined quite easily. Without the hole, the thickness of the layer of cool gas adjacent to the end wall is given by $\dagger$

$$
\delta=3 \cdot 6\left(\kappa_{5} t\right)^{\frac{1}{2}},
$$

where $\kappa_{5}$ is the thermal diffusivity in region (5) behind the reflected shock and $t$ is the time after shock reflexion. Two somewhat different cases arise depending on whether the orifice diameter $d$ is greater or less than $\delta$. In experiments at relatively high pressures $d \gg \delta$, and the flow into the orifice is inviscid (the 'ideal' case), while at lower pressures ( $d \ll \delta$, the 'classical' case) the sampled gas first diffuses through the thermal layer before entering the field of influence of the orifice about one diameter upstream of the end wall.

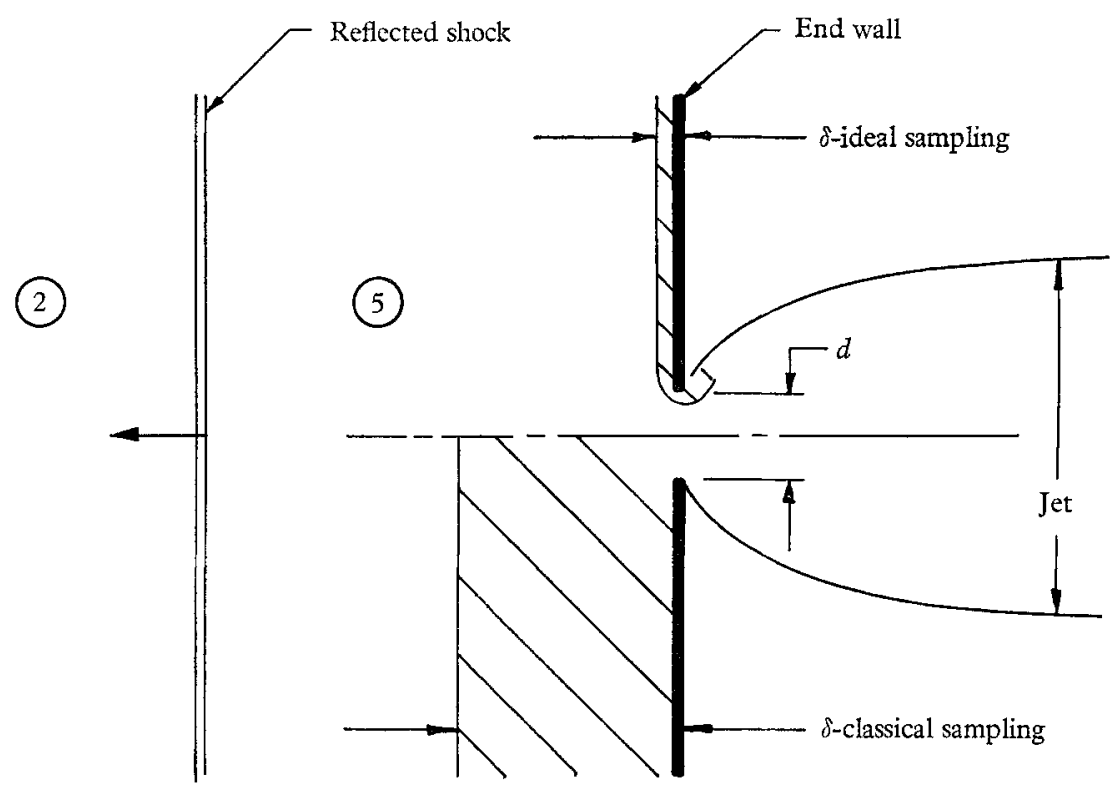

Figure 2. Flow field near the sampling orifice.

\subsection{The jet}

In either case, recent studies (Bier \& Hagena 1963; Fenn \& Deckers 1963) have shown that there are certain difficulties in generating a free-molecular beam from a high-pressure jet: for example, avoiding shock waves, or quasi-shocks in nearfree-molecular flow, is not a trivial matter. Such problems are alleviated (Fenn \& Deckers 1963) as the Knudsen number $\left(\mathrm{Kn}=\lambda^{*} / d\right.$, where $\lambda^{*}$ is the mean free path at, say, the sonic point in the orifice) is increased toward the free-molecular limit; in free-molecular effusion the expansion is the most 'rapid' in the sense used above. As will be shown below, the simplication of the fluid mechanics that is attendant on comparatively large $\mathrm{Kn}$ is a very important advantage of

$\dagger \delta$ is defined as the value of $x$ at which $T / T_{5}=0.99$ (see equations (4) and (5)). 
'classical' sampling. $\dagger$ However, it is important to note that the free-molecular limit, $\mathrm{Kn}>1$, can probably never be realized in shock-tube sampling experiments; for, if $K n>1$, then certainly $\lambda_{5} \gtrsim d$, but in all cases of interest $\lambda_{5} \ll \delta$, so $d \ll \delta$; i.e. the sampling is necessarily 'classical'. However, in all practical cases $\lambda^{*}$ in the very dense gas near the wall is smaller than the smallest orifices that can be made, so $\mathrm{Kn}<1$. For example, in the experiments reported in this paper $\lambda^{*} \approx 10^{-4} \mathrm{~cm}$.

Simple dimensional reasoning shows that the average number of collisions experienced by a particle in a jet that expands to free-molecular flow is

$$
N_{j}=A \mathrm{Kn}^{-1},
$$

where $A$ is a constant of order unity that depends on the jet geometry. In the 'ideal' case $N_{j}$ is very large because $d \gg \delta \gg \lambda_{5}$; the aim in sampling experiments is to reduce it as much as is practicable.

\subsection{The thermal layer}

On the other hand, the number of collisions a particle experiences while diffusing through the thermal layer is (see equation (14))

$$
N_{l l}=B t_{0} / \tau_{5}
$$

where $\tau_{5}=\lambda_{5} / \bar{c}_{5}$ is the mean collision time in region (5), and $t_{0}$ is the time at which the particle under consideration reaches the wall. Because $\delta \sim\left(\bar{c}_{5} \lambda_{5} t\right)^{\frac{1}{2}} \gg \lambda_{5}, N_{l l}$ is also very large, but is made as small as possible in 'classical' sampling experiments. In the 'classical' case $N_{j} / N_{l l} \approx \lambda_{5} d / \delta^{2} \ll 1$.

Comparison of equations (2) and (3) shows that the effect of lowering the pressure $p_{5}$ while keeping $d$ constant is to shift the region in which most of the degrading collisions occur from downstream of the orifice to upstream. If desired, this effect can be compensated by increasing $d$, but ultimately limitations on apparatus size become restrictive. For example, in the present experiments $\delta \approx 1 \mathrm{~cm}$, so for 'ideal' sampling $d \gg 1 \mathrm{~cm}$; attendant on the large pumping capacities implied by large $d$ or large $p_{5}$ (i.e. ideal sampling) is large apparatus size. On the other hand, if $\mathrm{Kn}$ is comparatively large, the distance downstream of the orifice to reach free molecular flow is shorter and so is the apparatus. This is another important advantage of 'classical' sampling.

We now proceed to calculate $N_{l l}$ for the present experiments. First, diffusion of charged particles in the thermal layert is described neglecting any production or depletion of species by chemical reactions. Then, the validity of this assumption is checked by calculating the number of collisions involving chemical change that an ion suffers in passing through the layer. The most important of such

$\dagger$ Evidently, the requirements for sampling are sufficiently different from those for generating collimated supersonic molecular beams (e.g. for studies of atomic collisions) that conclusions concerning the use of continuum nozzle expansions are somewhat different in the two cases.

† The analysis below is similar to that of Fay \& Kemp (1965), who studied heat transfer to the end wall. Here we restrict ourselves to consideration of slightly ionized gases, and examine the diffusion in somewhat more detail; the present analysis is applicable to any diffusion problem in which the mixture is dilute. 
collisions are charge exchange to an atom of a different species (exchange to an identical atom is not chemical change) and electron-ion recombination.

The charge density is sufficiently small that the gas temperature and density in the end-wall thermal layer are given by the well-known results for a perfect gas (Goldsworthy 1958). For example,

$$
\frac{T}{T_{1}}=1+\left(\frac{T_{5}}{T_{1}}-1\right) \operatorname{erf} \eta
$$

where $T_{1}$ is the wall and initial gas temperature and $\eta$, the boundary-layer co-ordinate, is

$$
\left.\begin{array}{rl}
\eta & =X / 2\left(\kappa_{5} t\right)^{\frac{1}{2}} \\
X & =\int_{0}^{x} \frac{\rho}{\rho_{5}} d x .
\end{array}\right\}
$$

The velocity induced by the accumulation of mass in the layer is

$$
v=-\left(\frac{\kappa_{5}}{\pi t}\right)^{\frac{1}{2}}\left(1-\frac{T_{1}}{T_{5}}\right)\left(1-e^{-\eta^{2}}\right) .
$$

The above expressions are derived under the assumption that the thermal conductivity is proportional to temperature,

$$
k / k_{5}=T / T_{5} .
$$

For the boundary condition on charge density at the wall, complete recombination, i.e. zero charge density at $x=0$, is assumed. Further, the charge density external to the boundary layer is taken to be independent of time, though this assumption can easily be relaxed since the equations to be considered are linear. The 'linearization' results from the further assumption of ambipolar diffusion, which replaces the non-linear electric coupling between ions and electrons with the condition of charge neutrality. An earlier investigation of electron-ion diffusion in an isothermal gas (Sturtevant 1961) showed that significant charge separation is preserved throughout the layer even at rather large $t$, but that, nevertheless, ambipolar diffusion can be used as an approximation to some aspects of the problem at very large times. (In the present experiments the Debye length in region (5) is about $10^{-3} \mathrm{~cm}$.) With this assumption, not only can the gas be treated as a binary mixture of ion-electron pairs and atoms (with diffusion coefficient twice the ion-atom coefficient) but, more important, a problem that has no inherent similarity properties (Sturtevant 1961) is reduced to one with similarity in the variable $\eta$.

It is also assumed that the Lewis number Le is constant, so equation (7) implies that the coefficient of diffusion is

$$
D=\operatorname{Le} \kappa_{5} \frac{T}{T_{5}} \frac{\rho_{5}}{\rho}
$$

Because of the large cross-section for charge exchange between ions and atoms of the parent gas, the diffusion coefficient and Le tend to be small; the value suggested by Fay \& Kemp (1965) for argon is Le $=0.25$.

The charged-particle continuity equation is

$$
n_{t}+[n(u+v)]_{x}=0
$$


where $u$ is the diffusion velocity; for a slightly ionized gas at constant pressure $\dagger$ (Hirschfelder, Curtiss \& Bird 1954),

$$
n u=-D\left(n_{x}+n T_{x} / T\right)
$$

Combining equations (9) and (10) and using the transformations (5) gives

$$
n^{\prime \prime}+n^{\prime}\left(\frac{2 \eta}{\mathrm{Le}}+3 \frac{T^{\prime}}{T}\right)-2 \frac{T^{\prime}}{T}\left(\frac{\mathrm{Le}-1}{\mathrm{Le}}\right) \eta n=0
$$

where the primes denote differentiation with respect to $\eta$ and equations (4) and (6) have been used to show that

$$
v^{\prime}\left(\kappa_{5} / t\right)^{-\frac{1}{2}}=-\eta T^{\prime} / T_{5}
$$

The boundary conditions are $n(0)=0$ and $n(\infty)=n_{5}$.

If, as in the present experiments, Le $<1$, it follows from equation (11) that $n(\eta)$ has a maximum, so $n>n_{5}$ in the outer part of the layer. This is simply because in this case the diffusion coefficient is sufficiently small that the convection of charged particles into the layer by the displacement velocity $v$ is larger than the rate at which they can diffuse to the wall. A similar, amplifying effect is caused by the fact that $D$ decreases by the factor $\left(T_{1} / T_{5}\right)^{2}$ through the layer, resulting in an important and drastic decrease of diffusion near the wall.

In figure 3 the distribution of argon ions calculated for the conditions of our experiment is plotted against $\eta$. Since the field of influence of the orifice extends about one diameter upstream, in effect the ions are sampled from the region $x \approx d$. The magnitude of $d$ at $t=100 \mu \mathrm{sec}$ in the experiments is indicated on figure 3. It is evident that the ion number density at the entrance to the orifice is of order $n_{5}$ and that, in fact, the thermal layer may act as an amplifier of charge density when Le is small!

\subsection{Reactions in the thermal layer}

Because the thermal layer is non-steady and non-uniform it is most convenient to consider its chemistry in terms of collision theory, by counting the number of collisions experienced by the ions occupying a volume element that moves through the thermal layer at the speed of an average diffusing ion. The average number of collisions an ion has in time $d t$ is

$$
d N_{l l}=\frac{\bar{c}}{\lambda} d t
$$

The rate at which the fluid particle progresses through the layer is $d x / d t=u+v$, which, with equations (5), implies a particle path $\eta(t)$ given by

$$
\ln \frac{t}{t_{0}}=-2 \int_{0}^{\eta(t)} \frac{d \eta}{\eta+\frac{1}{2} \operatorname{Le}\left\{\left(n^{\prime} / n\right)+\left(T^{\prime} / T\right)\right\}}
$$

$t_{0}$ is the time at which the particle reaches the wall. Combining equations (12) and (13) and making an assumption about the variation of $\lambda$ that is consistent with equation (7), namely $\lambda \sim T^{\frac{1}{2}} / \rho$,

$$
N_{l l}=2 \frac{\bar{c}_{5} t_{0}}{\lambda_{5}} \int_{0}^{\eta(\delta)} \frac{t / t_{0}}{T / T_{5}} \frac{d \eta}{\eta+\frac{1}{2} \operatorname{Le}\left\{\left(n^{\prime} / n\right)+\left(T^{\prime} / T\right)\right\}}
$$

$\dagger$ There is no thermal diffusion between species of equal mass. 


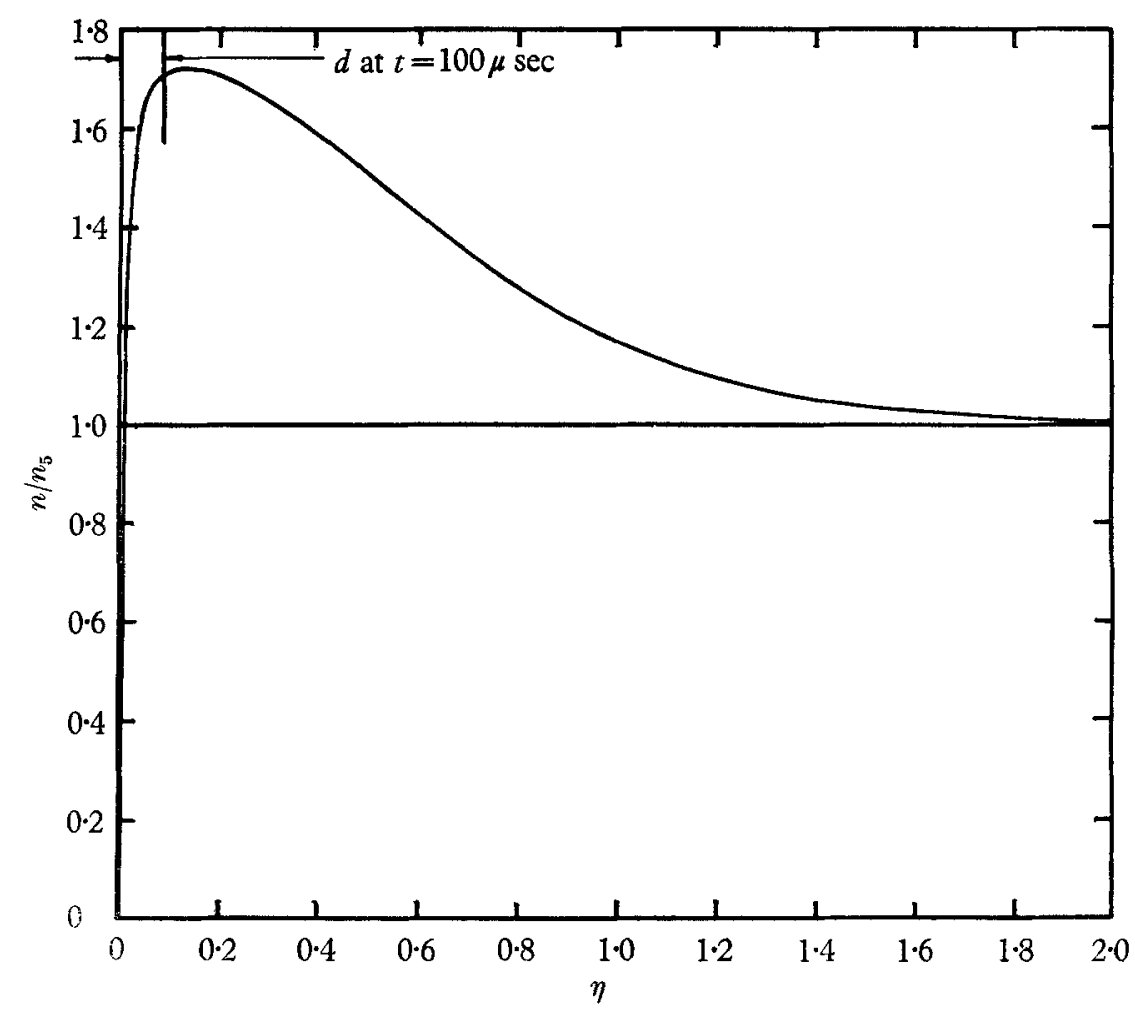

FIGURe 3. Charge distribution in the thermal layer for Le $=0 \cdot 25, T_{5} / T_{1}=55 \cdot 45$.

where $\eta(\delta)$ is the location near the edge of the thermal layer where $T$ departs significantly from $T_{5}$, e.g. $\eta=1.82$ for $T / T_{5}=0.99$ (equation (4)).

Various kinds of collisions in the thermal layer during the experiments in argon described in $\$ 4$ will now be considered using equation (14) with equation (13) and the solution to equation (11).

(a) $\mathrm{A}^{+}-\mathrm{A}$. The mean free path for collisions of argon ions with argon atoms can be computed from the charge-exchange cross-section, which Fay (1964) has estimated to be $130 \times 10^{-16} \mathrm{~cm}^{2}$; thus $\lambda_{5}=8 \cdot 7 \times 10^{-3} \mathrm{~cm}$. For Le $=0 \cdot 25$ the value of the integral in equation (14) is 1.57 . Thus, an argon ion that arrives at the wall at $t=100 \mu$ sec has had about 10,800 collisions not involving chemical change.

(b) $\mathrm{A}^{+}-\mathrm{I}$. Because charge exchange is a quantum-mechanical process and exhibits strong resonance, the charge-exchange cross-section is extremely small if the exchange energy in a collision is larger than a few tenths of an electron volt. For example, though for $\mathrm{A}^{+}-\mathrm{N}_{2}$ the exchange energy is only $0 \cdot 11 \mathrm{eV}$, the chargeexchange cross-section is probably an order of magnitude smaller than the elastic cross-section (Hasted 1952). Furthermore, in these experiments there are even very few elastic collisions between $A^{+}$and I; using as a conservative estimate the equivalent-hard-sphere cross-section for self-diffusion in argon, $13 \times 10^{-16} \mathrm{~cm}^{2}, \dagger$ and taking the highest impurity level encountered in these experiments $\left(3 \times 10^{-4}\right.$ impurity atoms per argon atom), $\lambda_{5}$ is calculated to be $290 \mathrm{~cm}$. In this case

$\dagger$ Calculated from the value of the diffusion coefficient computed by Amdur \& Mason (1958). 
$\mathrm{Le}=1$, and the value of the integral in equation (14) is 0.89 , so $N_{a}=0 \cdot 18$; less than 1 in 5 argon ions has an elastic collision with an impurity atom in the thermal layer.

(c) $\mathrm{I}^{+}-\mathrm{A}$. On the other hand, an impurity ion has about 610 elastic collisions with argon atoms in the thermal layer. However, except possibly for $\mathrm{N}_{2}^{+}-\mathrm{A}$, the charge-exchange cross-section is so small that loss of $\mathrm{I}^{+}$in the thermal layer is unlikely.

(d) $\mathrm{I}^{+-} \mathrm{I}$, Assuming that the cross-section for elastic $\mathrm{I}^{+}-\mathrm{I}$ collisions is about that used in $(b)$ above, less than 1 in $5 I^{+}$have an elastic collision with I. Similarly, the probability of charge exchange between different impurities in the thermal layer is probably small.

Other possible reactions in the thermal layer must be considered:

(e) Recombination. In the initial stages of ionization, when recombination in the shock-heated gas is negligible, recombination in the thermal layer tends to be small. In fact, a comparison of the loss of ions in the thermal layer due to threebody recombination with the gain due to transport into the layer $\dagger$ not only demonstrates that recombination in the layer is very small in the initial stages, but also that it is not even important in the xenon experiments described below until just before equilibrium is reached in region (5). The reason for this is that in these experiments the diffusive fluxes in the thermal layer are much larger than the reaction rates in the free stream.

( $f$ ) Ionization. The inelastic ionizing collisions that are of interest in region (5) do not occur in the thermal layer, because the strong temperature dependence of the Boltzmann factor, $\exp (-E / k T)$, that occurs in the rate equation for endothermic reactions ( $E$ is the activation energy) causes such reactions to 'freeze'; for $E=5 \mathrm{eV}$ and $k T=1 \mathrm{eV}$ (see $\S 5$ ) the rate is halved by a $15 \%$ decrease of temperature.

(g) The further possibility that impurities emitted from the wall during the experiment may enter the thermal layer in sufficient numbers to affect the above considerations may be discounted because the degassing rate implied by the measured 'leak rate' of this shock tube is roughly eight orders of magnitude smaller than the diffusive flux from the shock-heated gas into the layer (e.g. $n_{5} v(\delta)$ ).

Thus, it is concluded that in these experiments the products of the ionization reactions in the hot gas behind the reflected shock wave will not be greatly changed by their passage through the thermal layer and that the ions detected by the mass spectrometer are those produced by ionizing collisions in the shockheated gas.

\section{Experiments}

In this section two experiments are described: first, a test of the experimental technique and apparatus by measuring the approach to equilibrium ionization in xenon, and, secondly, the primary objective of this paper, an investigation of the early stages of ionization in argon.

$\uparrow$ The ratio of these two quantities is of order $k_{R} n_{5} t$, where $k_{R}$ is the recombination coefficient in the thermal layer obtained from Massey \& Burhop (1952). 


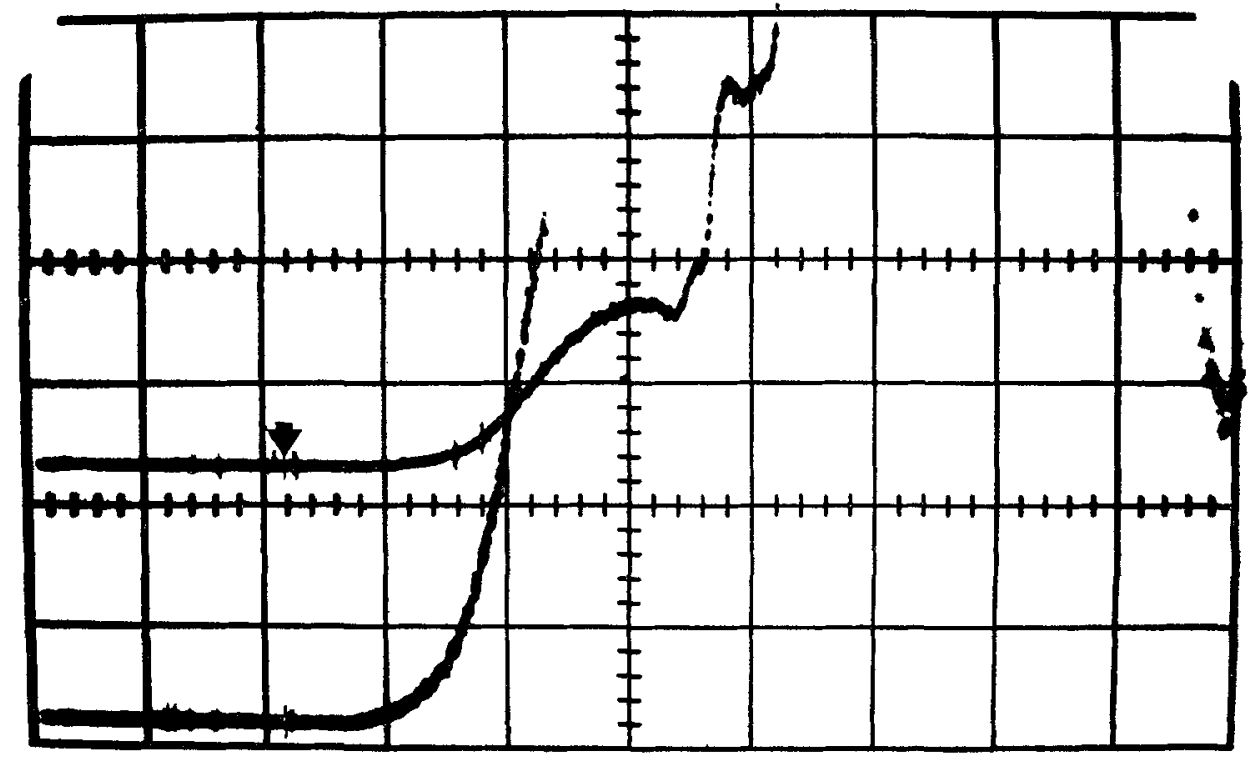

Figure 4. Mass spectrometer output against time in xenon. $M_{s}=9 \cdot 1, p_{1}=30 \mu \mathrm{Hg}$. Horizontal scale $100 \mu$ sec per large division; vertical scale, upper beam $0.020 \mathrm{~V}$ per large division, lower beam $0.005 \mathrm{~V}$ per large division.

\subsection{Xenon}

With the mass spectrometer set on mass-peak 129 it was found that for $M_{s}=9 \cdot 1$ and $p_{1}=30 \mu \mathrm{Hg}$ (equilibrium ionization $12 \%, T_{5}=7700^{\circ} \mathrm{K}$ ) equilibrium was reached just before the run was terminated by the arrival of the shock wave reflected from the contact surface (figure 4). The arrow on the upper trace of figure 4 indicates the time at which the shock reflected from the end wall, i.e. $t=0$; the relaxation time implied by the trace is about $300 \mu \mathrm{sec}$. If Turner's data for relaxation times behind incident shock waves, as reported by Blackman $\&$ Niblett (1961), are extrapolated to the much higher temperatures of the present experiment, they imply a relaxation time of $80 \mu \mathrm{sec}$. In view of the fact that Turner, in the original reference, stated that a different, purer sample of gas gave relaxation times five times larger than those reported by Blackman \& Niblett, it can be concluded that the interpretation of the trace in figure 4 as being an accurate record of the ionization process is correct. This result demonstrates that the sampling technique can be used for accurate measurements of many features of ionization processes.

\subsection{Argon}

In this experiment the initial pressure in the shock tube was $30 \mu \mathrm{Hg}$ and in the 17 runs from which the time-resolved mass spectrum was obtained the incident shock Mach number averaged $8.56 \pm 0.06$ (r.m.s. deviation). Corresponding to these conditions, the perfect-gas pressure and temperature behind the reflected shock wave were $15 \cdot 7 \mathrm{mmHg}$ and $16,640^{\circ} \mathrm{K}$ respectively, and the relaxation time for ionization in region (5) was probably about 2 msec (Petschek \& Byron 1957). The average estimated impurity level was 300 parts per million, and the calculated testing time was $176 \mu$ sec. 


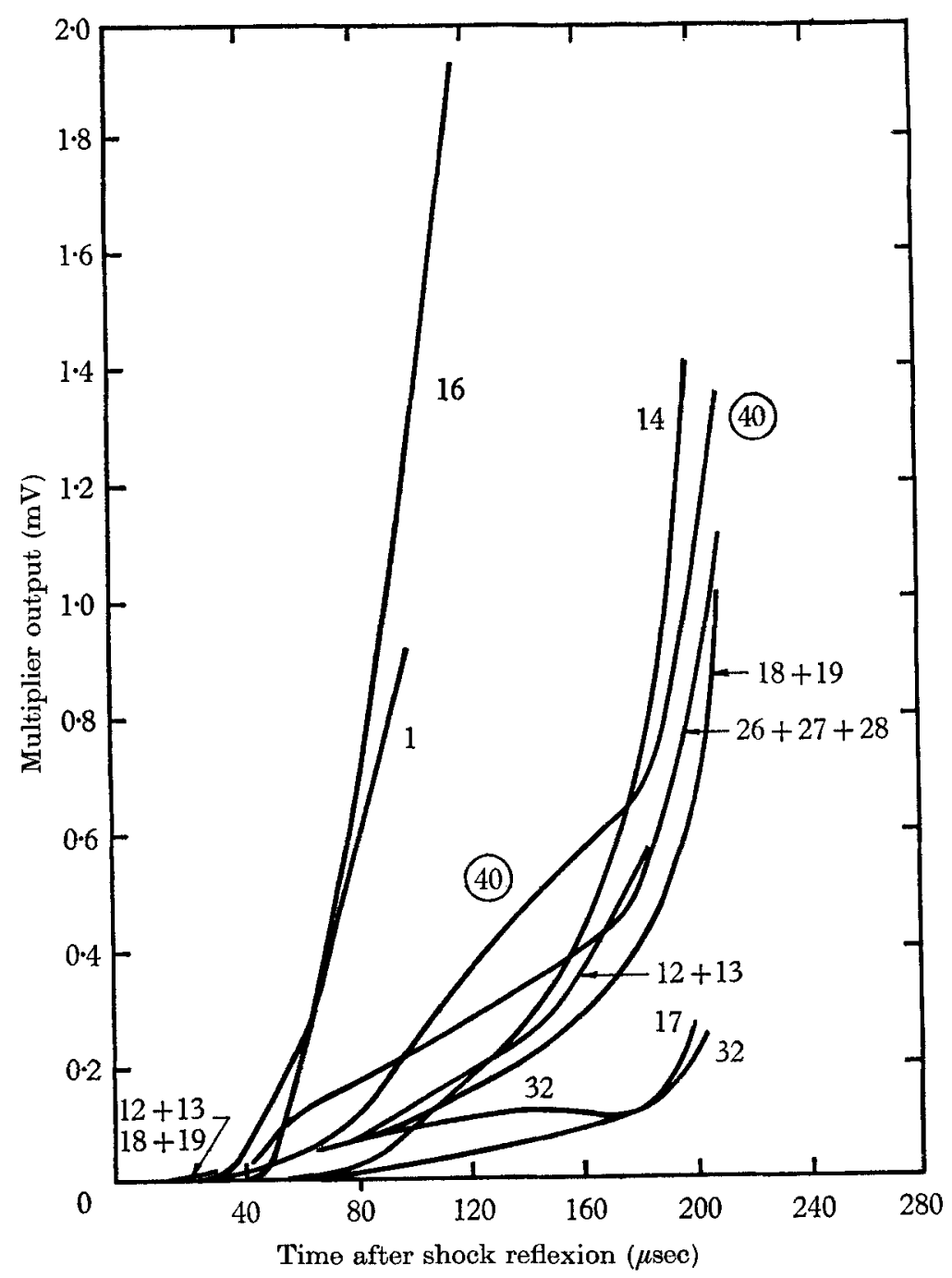

Figure 5. Mass spectrometer output against time for various mass peaks in argon. Conditions: $M_{5}=8 \cdot 56, p_{1}=30 \mu \mathrm{Hg}, T_{5}=16,640^{\circ} \mathrm{K}, p_{5}=15.7 \mathrm{mmHg}$.

The mass spectrometer output for these experiments is plotted against time in figure 5. A surprisingly large number of different species was observed; of all the mass peaks that were investigated, only $m=2,15$ and 44 gave no discernible signal. Qualitatively, the traces look very much like those in our earlier measurements, under somewhat different conditions, of the total electric current through a sampling orifice (Sturtevant 1961); $\dagger$ the ionization starts after a sizeable incubation period $\ddagger$ and initially rises quite non-linearly.

† In the present experiments the total current also was measured and was found to be very nearly equal to the sum of the individual currents.

$\ddagger$ The time of flight in the mass spectrometer is only between 1 and $7 \mu$ sec. 
The most important feature of figure 5 is the occurrence of $\mathrm{O}^{+}$and $\mathrm{H}^{+}$in much greater amounts $t_{\text {than }}$ any of the other products, each being five times more abundant than $\mathrm{A}^{+}$, for example. $\ddagger$ Presumably, the major source of $\mathrm{O}^{+}$and $\mathrm{H}^{+}$is water vapour, which, after $\mathrm{N}_{2}$, is the most abundant component of the residual gas at ultimate vacuum in our shock tube. Why the ionization of $\mathrm{O}$ and $\mathrm{H}$ is so efficient is the subject of $\S 5$.

\section{Discussion}

Because the components of the residual gas in all reasonably tight, unbakeable vacuum systems are substantially the same, it is likely that the important impurity reactions and products in other shock-tube ionization experiments will be found to be substantially the same as in this study. All indications are that the most important reactants are atomic species. Consequently, the occurrence of the incubation period can probably be ascribed to the fact that molecular impurities, in particular $\mathrm{H}_{2} \mathrm{O}$, must dissociate before ionization commences. 'This conclusion is substantiated by the fact that $\mathrm{H}_{2} \mathrm{O}^{+}$is the first product to appear in figure 5 (at $t=20 \mu \mathrm{sec}$ ), but, shortly after, $\mathrm{O}^{+}$and $\mathrm{H}^{+}$completely dominate. Evidence of other, slower dissociation processes also appears in figure 5: the fact that the output from $m=32$ seems to have a maximum at $t=150 \mu \mathrm{sec}$ could be caused by the dissociation of $\mathrm{O}_{2}$, and the $m=14$ trace, which has one of the largest incubation times, eventually becomes much larger than the $m=28$ trace.

In order to account for the observed abundance of $\mathrm{O}^{+}$and $\mathrm{H}^{+}$, in view of the fact that the corresponding atomic species are estimated to be in concentrations of less than $3 \times 10^{-4}$, the mechanism for ionization must be associated with either a very large cross-section or a very small activation energy. Indeed, estimates from previous measurements of ionization rates (e.g. Kelly 1965) suggest that if reasonable cross-sections are assumed the impurity activation energy must be of order $5 \mathrm{eV}$. With this and the meagre information already obtained from the mass spectrometer, it is possible to speculate on possible mechanisms; it appears that the ionization of $\mathrm{O}$ is associated with a low activation energy and the ionization of $\mathrm{H}$ with a large cross-section.

\subsection{Oxygen ionization}

$\mathrm{C}, \mathrm{N}$ and $\mathrm{O}$ are distinguished among the light, common elements by the fact that they have metastable electronic states at energy levels intermediate between the ground and first-excited states. Since the radiative lifetime of these states is very long and the energy gaps between adjacent states are relatively small, it is possible that they can be collisionally populated and play an important role as

$\dagger$ The possibility that $\mathrm{CH}_{4}^{+}$accounts for the $m=16$ output is ruled out by several arguments, not the least of which is that at these temperatures the pyrolysis of methane should be very rapid.

‡ Additional experiments with an estimated impurity level between ten and thirty times less than that of figure 5 (in which the possibility of chemical change in the thermal Iayer is drastically reduced) give qualitatively the same results, $m=16$ being about three times more abundant than $m=40$. 
intermediate states in the ionization process. If, as is usually the case, the transition to a particular one of the states from the next lower state is rate-determining, all other transitions being very rapid and the corresponding states being in quasi-equilibrium, the apparent activation energy for ionization will be that of the rate-determining step. The largest energy gap between any two adjacent metastable states of $\mathrm{O}$ is smaller than that of $\mathrm{C}$ or $\mathrm{N}$; its metastable states are $1.95 \mathrm{eV}\left({ }^{1} D\right), 4.7 \mathrm{eV}\left({ }^{1} S\right)$ and $9 \cdot 15 \mathrm{eV}\left(3 s^{5} S\right.$ subresonance state) above the ground state, and the corresponding energy differences are $1.95,2.22$ and $4.98 \mathrm{eV}$, leaving a $4.46 \mathrm{eV}$ jump to the continuum at $13 \cdot 61 \mathrm{eV}$. Thus, if the ionization of $O$ is due to a many-step process and the transition involving the largest energy difference is the rate-determining step, the apparent activation energy should be of order $5 \mathrm{eV}$.

\subsection{Hydrogen ionization}

If charge exchange occurs between two different atoms with nearly identical ionization potentials, a fortuitous energy balance occurs and the cross-section for the process tends to be very large. Such a phenomenon is known as accidental resonance. One such case, electron exchange from $\mathrm{H}$ to $\mathrm{O}^{+}$with reactants and products in ground states, has been studied experimentally by Fite, Smith \& Stebbings (1962). The energy balance in this case depends on which level of the triplet ground state of atomic oxygen is involved:

$$
\left.\mathrm{O}^{+}\left({ }^{4} S\right)+\mathrm{H}(1 s) \rightarrow \mathrm{O}^{3} P_{J}\right)+\mathrm{H}^{+}\left\{\begin{array}{l}
-0.01 \mathrm{eV}(J=0) \\
+0.00 \mathrm{eV}(J=1) \\
+0.02 \mathrm{eV}(J=2)
\end{array}\right\}
$$

The nature of the process for the case $\Delta E=0$ is a matter of some controversy. For $\Delta E \neq 0$ the cross-section is maximum at collision velocities $v$ for which the collision time $a / v$, where $a$ is the spatial extent of the interaction force field, is equal to the inverse of the charge-exchange frequency $\Delta E / h$. Hasted $(1952,1965)$ has found that the value $a=7 \AA$ correlates the maxima of virtually all experimentally measured charge-exchange cross-sections. Taking $v$ to be the mean relative collision velocity in region (5) in the present experiments, and

$$
\text { the adiabatic parameter is } \quad \begin{aligned}
& \Delta E=0.01 \mathrm{eV}, \\
& \overline{v_{r}} h / a \Delta E \doteq 1.1 .
\end{aligned}
$$

Therefore, these experiments were made at just the temperature at which the cross-section for $\mathrm{O}^{+}-\mathrm{H}$ charge exchange should be maximum (probably several times larger than the corresponding gas-kinetic cross-section). It is conceivable, therefore, that the $\mathrm{H}^{+}$observed in figure 5 is in fact due to reaction (15).

\section{Conclusion}

An inexpensive magnetic mass spectrometer for sampling ions from shockheated gas near the end wall of a shock tube has been described. Sampling through the end-wall thermal layer has been analysed in some detail, and it has been shown that this mode of operation, when possible, offers a relatively simple fluid-mechanical field in which chemical changes caused by the sampling process can be estimated. Furthermore, the experimental apparatus is smaller and 
simpler than if an inviscid nozzle expansion were used for sampling, and the sampled species suffer most of their collisions at low temperatures rather than in more chemically active high-temperature gas.

Analysis of the ions sampled from 'impure' shock-heated argon during the initial stages of ionization relaxation has shown that $\mathrm{O}^{+}$and $\mathrm{H}^{+}$are the most abundant products. This result suggests some intriguing explanations of the well-known ionization efficiency of relatively small amounts of impurity.

Further experiments at different thermodynamic conditions are under way. Now that the reaction products are known, ionization of these impurities mixed in known amounts with inert gases can be studied to obtain information on the nature of the mechanism. For this purpose, it would be useful if, in addition to making the purely qualitative analysis reported here, the sampling technique could be used for measuring rates, etc. Though this was not originally contemplated, the prospects for such a development, in the light of the present study, are encouraging. One possibly interesting question that remains to be investigated is what the ionization relaxation time of the impurities in the argon is, and what happens after impurity ionization has reached equilibrium.

The author wishes to thank Mr Charles $\mathrm{P}$. Wang for his important contributions to the design of the electrical circuits, construction of the instrument, and execution of the experiments. This work was supported by NASA Grant NsG 40-60.

\section{REFERENCES}

Amdur, I. \& Mason, E. A. 1958 Phys. Fluids, 1, 370.

Bier, K. \& Hagena, O. 1963 Rarefied Gasdynamics, p. 478 (ed. J. Laurmann). Now York: Academic Press.

Blackman, V. H. \& Niblett, G. B. F. 1961 Fundamental Data Obtained from Shock-Tube Experiments, p. 221 (ed. A. Ferri). New York: Pergamon Press.

Bradify, J. N. \& Kistrakowsky, G. B. 1961 J. Chem. Phys. 35, 256.

FAY, J. A. 1964 The High Temperature Aspects of Hypersonic Flow, p. 590 (ed. W. C. Nelson). New York: Pergamon Press.

FAY, J. A. \& KEMP, N. H. 1965 J. Fluid Mech. 21, 659.

Fen, J. B. \& Deckers, J. 1963 Rarefied Gasdynamics, p. 497 (ed. J. Laurmann). New York: Academic Press.

Fite, W. L., Smith, A. C. H. \& Stebbinas, R. F. 1962 Proc. Roy. Soc. A, $268,527$.

Goldsworthy, F. A. 1958 J. Fluid Mech. 5, 1964.

Harweld, K. E. \& J.AHN, R. G. 1964 Phys. Fluids, 7, 214. Corrigendum p. 1554.

Hasted, J. B. 1952 Proc. Roy. Soc. A, 212, 235.

HASTED, J. B. 1965 Physics of Atomic Collisions, p. 421. London: Butterworths.

HirschferdDer, J. O., CURTiss, C. F. \& BIRD, R. B. 1954 Molecular Theory of Gases and Liquids, p. 518. New York: John Wiley and Sons, Ine.

KeLIY, A. J. 1965 Private communication.

Lmepmane, H. W., Roshko, A., Coles, D. \& Sturtevant, B. 1962 Rev. Sci. Instr. 33, 625.

Massey, H. S. W. \& BURHoP, E. H. S. 1952 Electronic and Ionic Impact Phenomena, p. 625. Oxford University Press.

NIER, A. O. 1940 Rev. Sci. Instr. 11, 212.

Nier, A. O, 1947 Rev. Sci. Instr. 18, 398.

Petschex, A. \& Bxron, S. 1957 Ann. Phys. (N.Y.), 1, 270.

Sturtevant, B. 1961 Phys. Fluids, 4, 1064. 